\title{
ON CO-RECURSIVE ORTHOGONAL POLYNOMIALS
}

\section{T. S. CHIHARA}

1. Introduction. Let $P_{n}(x)$ be a polynomial of degree $n(n=0,1, \cdots)$ with leading coefficient unity. Then (Favard [2]) a sufficient (as well as necessary) condition for the $P_{n}(x)$ to be real orthogonal polynomials is that they satisfy a recurrence formula of the form

$$
P_{n}(x)=\left(x+b_{n}\right) P_{n-1}(x)-\lambda_{n} P_{n-2}(x), \quad n=2,3, \cdots
$$

where $P_{0}(x)=1, P_{1}(x)=x+b_{1}, b_{n}$ real, $\lambda_{n}>0$.

D. Dickinson [1] has shown that a relation of the form

$$
\begin{aligned}
P_{n}(x) P_{n-1}(-x)+P_{n}(-x) P_{n-1}(x)=a_{n},(-1)^{n} a_{1} a_{n} & <0, \\
n & =1,2, \cdots,
\end{aligned}
$$

is equivalent to (1.1) with $b_{n}=0(n \geqq 2)$ and $P_{1}(0) \neq 0$. The condition $b_{n}=0(n \geqq 2)$ suggests the symmetric case, (i.e., $\left.P_{n}(-x)=(-1)^{n} P_{n}(x)\right)$ but this is denied by the condition $P_{1}(0) \neq 0$. (In fact, (1.2) shows that $P_{n}(-r) \neq 0$ whenever $P_{n}(r)=0$.) It then seems natural to ask what relations exist between a set of polynomials satisfying (1.2) and the corresponding symmetric polynomials which would be obtained from the equivalent relation (1.1) if the condition $P_{1}(0) \neq 0$ were replaced with $P_{1}(0)=0$.

We are thus led to consider the following more general situation. Given the $P_{n}(x)$ satisfying (1.1) (without the restriction $b_{n}=0$ ), let the "co-recursive" polynomials $P_{n}^{*}(x) \equiv P_{n}^{*}(x, c)$ be defined by

$$
P_{n}^{*}(x)=\left(x+b_{n}\right) P_{n-1}^{*}(x)-\lambda_{n} P_{n-2}^{*}(x), \quad n=2,3, \cdots
$$

where $P_{0}^{*}(x)=1, P_{1}^{*}(x)=P_{1}(x)-c$. We then seek to determine the properties of the $P_{n}^{*}(x)$ from those of the $P_{n}(x)$. In order to have orthogonal polynomials, we will restrict $c$ to be real.

2. Some formal relations. Since $P_{n}(x)$ and $P_{n}^{*}(x)$ satisfy identical recurrence formulas, except for initial conditions, it follows that $P_{n}^{*}(x)-P_{n}(x)$ also satisfies (1.1) together with the initial conditions, $P_{0}^{*}(x)-P_{0}(x)=0, P_{1}^{*}(x)-P_{1}(x)=-c$. Therefore we have ${ }^{1}$

Presented to the Society, December 29, 1956; received by the editors January 2, 1957 and, in revised form, February 19, 1957.

1 Sherman [3] noted a relation of this type for certain orthogonal polynomials associated with a Stieltjes continued fraction. 


$$
P_{n}^{*}(x)=P_{n}(x)-c Q_{n-1}(x), \quad n=0,1, \cdots
$$

where $Q_{n}(x)$ is a polynomial of degree $n$, satisfying

$$
Q_{n}(x)=\left(x+b_{n+1}\right) Q_{n-1}(x)-\lambda_{n+1} Q_{n-2}(x), \quad n=1,2, \cdots
$$

where $Q_{-1}(x)=0, Q_{0}(x)=1$.

Comparison of the recurrence formulas shows that $Q_{n-1}(x) / P_{n}(x)$ and $Q_{n-1}(x) / P_{n}^{*}(x)$ are the $n$th convergents, respectively, of the continued fractions

$$
\begin{aligned}
K(z) & =\frac{1 \mid}{\mid z+b_{1}}-\frac{\lambda_{2} \mid}{\mid z+b_{2}}-\frac{\lambda_{3} \mid}{\mid z+b_{3}}-\cdots \\
K^{*}(z) & =\frac{1 \mid}{\mid z+b_{1}-c}-\frac{\lambda_{2} \mid}{\mid z+b_{2}}-\frac{\lambda_{3} \mid}{\mid z+b_{3}}-\cdots
\end{aligned}
$$

The $Q_{n}(x)$ are the "numerator polynomials" studied by Shohat and Sherman [4] and Sherman [3]; $Q_{n}(x)$ is itself the denominator of the $n$th convergent of the continued fraction

$$
K_{1}(z)=\frac{1 \mid}{\mid z+b_{2}}-\frac{\lambda_{3} \mid}{\mid z+b_{3}}-\frac{\lambda_{4} \mid}{\mid z+b_{4}}-\cdots
$$

We will denote by $\psi(x), \psi^{*}(x) \equiv \psi^{*}(x, c)$ and $\psi_{1}(x)$, respectively, solutions of the moment problems associated with the above continued fractions and write $F(z), F^{*}(z)$ and $F_{1}(z)$ for the corresponding Stieltjes transforms:

$$
K(z) \sim F(z)=\int_{-\infty}^{\infty}(z-u)^{-1} d \psi(u)(z \text { nonreal }), \text { etc. }
$$

3. Zeros. By Favard's theorem, the sets $\left\{P_{n}(x)\right\},\left\{P_{n}^{*}(x)\right\}$ and $\left\{Q_{n}(x)\right\}$ are orthogonal with respect to the distributions $\psi(x), \psi^{*}(x)$ and $\psi_{1}(x)$, respectively; hence the zeros of the individual polynomials are real and simple.

If we multiply (1.1) by $P_{n-1}^{*}(x)$ and (1.3) by $P_{n-1}(x)$ and subtract, we obtain

$P_{n-1}^{*}(x) P_{n}(x)-P_{n}^{*}(x) P_{n-1}(x)=\lambda_{n}\left[P_{n-2}^{*}(x) P_{n-1}(x)-P_{n-1}^{*}(x) P_{n-2}(x)\right]$.

Iterating this relation yields

$$
P_{n}^{*}(x) P_{n-1}(x)-P_{n-1}^{*}(x) P_{n}(x)=-c \prod_{k=1}^{n} \lambda_{k} \quad\left(n \geqq 1, \lambda_{1}=1\right) .
$$

If $x_{n, j}(j=1, \cdots, n)$ denotes the zeros of $P_{n}(x)$ in ascending order of magnitude, then $P_{n}^{*}\left(x_{n, j}\right) P_{n-1}\left(x_{n, j}\right)=-c \prod_{k=1}^{n} \lambda_{k}$, so $P_{n}^{*}\left(x_{n, j}\right)$ 
alternates in sign with $P_{n-1}\left(x_{n, j}\right)$. By the well known separation of the zeros of consecutive orthogonal polynomials, it follows that the zeros of $P_{n}(x)$ and $P_{n}^{*}(x)$ are mutually separated. Moreover, $P_{n-1}\left(x_{n, n}\right)$ $>0$, hence $\operatorname{sgn} P_{n}^{*}\left(x_{n, n}\right)=-\operatorname{sgn} c$. Therefore if $x_{n, j}^{*}(j=1, \cdots, n)$ denotes the zeros of $P_{n}^{*}(x)$ in ascending order of magnitude, we have

$$
x_{n, j-1}<x_{n, j-1}^{*}<x_{n, j}<x_{n, j}^{*} \quad(j=2, \cdots, n ; c>0),
$$

with the roles of $x_{n, j}$ and $x_{n, j}^{*}$ interchanged for $c<0$.

Finally, let $x_{n-1, j}^{\prime} \equiv y_{n, j}(j=1, \cdots, n-1)$ denote the zeros of $Q_{n-1}(x)$ in ascending order. Then it is well known (e.g. [6]) that

$$
x_{n, j-1}<y_{n, j-1}<x_{n, j} ; \quad x_{n, j-1}^{*}<y_{n, j-1}<x_{n, j}^{*}(j=2, \cdots, n) .
$$

Combining (3.1) and (3.2), we have

Theorem 1. The zeros of $P_{n}(x), P_{n}^{*}(x, c)$ and $Q_{n-1}(x)(n \geqq 2)$ are mutually separated in the following manner:

$$
x_{n, j-1}<x_{n, j-1}^{*}<y_{n, j-1}<x_{n, j}<x_{n, j}^{*} \quad(j=2, \cdots, n ; c>0),
$$

with the roles of $x_{n, j}$ and $x_{n, j}^{*}$ reversed for $c<0$.

COROLlaRY. The zeros of $P_{n}^{*}(x, c)$ are increasing functions of $c$ and the zeros of any two distinct co-recursive orthogonal polynomials of the same degree $n(n \geqq 1)$ are mutually separated.

Proof. Let $P_{n}^{*}\left(x, c_{1}\right)$ and $P_{n}^{*}\left(x, c_{2}\right)$ be any two-recursive polynomials. Let $P_{n}^{*}\left(x, c_{1}\right)=P_{n, 1}(x)$ and $P_{n}^{*}\left(x, c_{2}\right)=P_{n, 2}(x)$. Then $P_{n, 2}\left(x, c_{1}-c_{2}\right)=P_{n, 1}(x)$.

Next let $[a, b],\left[a^{*}, b^{*}\right]$ and $\left[a^{\prime}, b^{\prime}\right]$ denote the "true" intervals of orthogonality of $\left\{P_{n}(x)\right\},\left\{P_{n}^{*}(x)\right\}$ and $\left\{Q_{n}(x)\right\}$, respectively; that is [4], $a=\lim _{n \rightarrow \infty} x_{n, 1}, b=\lim _{n \rightarrow \infty} x_{n, n}$, etc. Then by Theorem 1 , for $c>0, a \leqq a^{*} \leqq a^{\prime}<b^{\prime} \leqq b \leqq b^{*}$, and for $c<0, a^{*} \leqq a \leqq a^{\prime}<b^{\prime} \leqq b^{*} \leqq b$. Further, $a=-\infty(b=+\infty)$ if and only if $a^{\prime}=-\infty\left(b^{\prime}=+\infty\right)$ [3], hence $a, a^{*}, a^{\prime}\left(b, b^{*}, b^{\prime}\right)$ are all finite or all infinite. If $a$ or $b$ is finite, Theorem 1 shows that $P_{n}^{*}(x)$ has at most one zero exterior to $[a, b]$, while all zeros of $Q_{n}(z)$ (for all $n$ ) are interior to $[a, b]$.

THEOREM 2. The zeros of $P_{n}^{*}(x, c)$ are all interior to $[a, b]$ for all $n$ if and only if

$$
\lim _{n \rightarrow \infty} \frac{P_{n}(a)}{Q_{n-1}(a)} \equiv A \leqq c \leqq B \equiv \lim _{n \rightarrow \infty} \frac{P_{n}(b)}{Q_{n-1}(b)} .
$$

Here $A(B)$ must be replaced by $-\infty(+\infty)$ in case $a=-\infty(b=+\infty)$. 
Proof. Suppose $a$ is finite. Then it follows from the "determinant formula" for continued fractions [6] that for $x \leqq a, P_{n}(x) / Q_{n-1}(x)$ $<P_{n+1}(x) / Q_{n}(x)<0$ (as is known). Hence $A$ exists and by (2.1)

$$
P_{n}^{*}(a) / Q_{n-1}(a)=P_{n}(a) / Q_{n-1}(a)-c<A-c \quad(n \geqq 2) .
$$

Thus $P_{n}^{*}(a) / Q_{n-1}(a)<0$ and $P_{n}^{*}(x)$ does not change sign for $x \leqq a$ (for all $n$ ) if and only if $A \leqq c$.

4. On the distribution functions. Sherman [3] has shown that the complete convergence of (2.3) implies the complete convergence of (2.5). By a theorem of Carleman (cf. [5]) or by direct verification, the complete convergence of (2.3) is seen to imply the complete convergence of (2.4) also. Thus if $\psi(x)$ is a solution of a determined moment problem, and hence is uniquely determined up to an additive constant at all points of continuity [5], then so is $\psi^{*}(x)$ (as well as $\left.\psi_{1}(x)\right)$. Moreover, if we define

$$
\begin{aligned}
& q_{n}(x)=\left(\lambda_{1} \cdots \lambda_{n+1}\right)^{-1 / 2} Q_{n-1}(x), \quad p_{n}(x)=\left(\lambda_{1} \cdots \lambda_{n+1}\right)^{-1 / 2} P_{n}(x), \\
& p_{n}^{*}(x)=\left(\lambda_{1} \cdots \lambda_{n+1}\right)^{-1 / 2} P_{n}^{*}(x), \\
& n=0,1,2, \cdots,
\end{aligned}
$$

then $\sum_{n=0}^{\infty}\left|p_{n}(x)\right|^{2}$ diverges at all points of continuity of $\psi(x)$ and converges at a discontinuity of $\psi(x)$ [5]. Similar interpretations hold for $\sum_{n=0}^{\infty}\left|p_{n}^{*}(x)\right|^{2}$ and $\sum_{n=0}^{\infty}\left|q_{n}(x)\right|^{2}$. But if (2.3) corresponds to a determined moment problem, then $\sum_{n=0}^{\infty}\left|p_{n}(x)\right|^{2}$ and $\sum_{n=0}^{\infty}\left|q_{n}(x)\right|^{2}$ cannot converge for a common value of $x$ [7]. It follows from (2.1) that no two of the above three series can converge for a common value of $x$, hence we have

THEOREM 3. If $\psi(x)$ is a solution of a determined moment problem, then so are $\psi^{*}(x)$ and $\psi_{1}(x)$. In this case, no two of the three distribution functions can have a common discontinuity.

The above argument shows that if, say, $b$ is finite and $B \neq 0$, then $\sum_{n=0}^{\infty}\left|p_{n}(b)\right|^{2}$ and $\sum_{n=0}^{\infty}\left|q_{n}(b)\right|^{2}$ must diverge together, hence $\psi(x)$ and $\psi_{1}(x)$ must be continuous at $b$. Similarly, $\psi^{*}(x)$ is continuous at $b$ if $c \neq B$.

Henceforth, we assume (2.3) corresponds to a determined moment problem so that by $[3]$, the continued fractions $(2.3),(2.4)$ and (2.5) converge completely for all nonreal $z$ to $F(z), F^{*}(z)$ and $F_{1}(z)$, respectively, with $F(z)=\left[z+b_{1}-\lambda_{2} F_{1}(z)\right]^{-1}$ and $F^{*}(z)=\left[z+b_{1}-c-\lambda_{2} F_{1}(z)\right]^{-1}$. Also,

$$
F^{*}(z)=F(z) /[1-c F(z)]
$$


We further assume that $\psi(x), \psi^{*}(x)$ and $\psi_{1}(x)$ have been normalized: $\psi(x)=[\psi(x+0)+\psi(x-0)] / 2$, etc. Then by the Stieltjes inversion formula

$$
\psi^{*}(x)-\psi^{*}\left(x_{0}\right)=\lim _{\nu \rightarrow 0^{+}} \frac{1}{\pi} \int_{x}^{x_{0}} \operatorname{Im} \frac{F(u+i y)}{1-c F(u+i y)} d u .
$$

Sherman [3] has shown that if the analytic continuation of $F(z)$ is such that $1 / F(z)$ is regular on a segment, $\left[x_{0}, x\right]$, of the real axis, then

$$
\psi_{1}(x)-\psi_{1}\left(x_{0}\right)=\frac{1}{\pi} \int_{x}^{x_{0}} \operatorname{Im}[F(u)]^{-1} d u .
$$

If the analytic continuation of $F^{*}(z)$ is regular on $\left[x_{0}, x\right]$, then by the same procedure used by Sherman, we can show that

$$
\psi^{*}(x)-\psi^{*}\left(x_{0}\right)=\frac{1}{\pi} \int_{x}^{x_{0}} \operatorname{Im} \frac{F(u)}{1-c F(u)} d u .
$$

If $\psi(x)$ has an interval of constancy, $(\alpha, \beta)$, then (2.6) defines a single analytic function which is regular on the upper and lower halfplanes and $(\alpha, \beta)$. If $F(\xi)=1 / c, \alpha<\xi<\beta$, then $F^{*}(z)$ has a pole at $\xi$ and, in general, $\psi^{*}(x)$ will have a jump at $\xi$ which can be determined as follows.

We can choose $x_{0}<\xi<x$ so that $\left[x_{0}, x\right] \subset(\alpha, \beta)$ and $F(u) \neq 1 / c$ for $u \neq \xi, u \in\left[x_{0}, x\right]$. On the closed rectangular contour $\gamma$, whose vertices are $x+i y, x_{0}+i y, x_{0}-i y, x-i y(y>0)$, we have by the residue theorem

$$
\begin{aligned}
& \int_{x}^{x_{0}}\left[F^{*}(u+i y)-F^{*}(u-i y)\right] d u+i \int_{-y}^{y}\left[F^{*}(x+i v)\right.\left.-F^{*}\left(x_{0}+i v\right)\right] d v \\
&=2 \pi i \operatorname{Res}_{z=\xi} F^{*}(z), \\
& \frac{1}{\pi} \int_{x}^{x_{0}} \operatorname{Im} F^{*}(u+i y) d u \\
&=\operatorname{Res}_{z=\xi} F^{*}(z)+\frac{1}{\pi} \int_{0}^{y} \operatorname{Re}\left[F^{*}(x+i v)-F^{*}\left(x_{0}+i v\right)\right] d v .
\end{aligned}
$$

Since $F^{*}(z)$ is bounded on $\gamma$, we obtain on letting $y \rightarrow 0+$,

$$
\psi^{*}(x)-\psi^{*}\left(x_{0}\right)=\operatorname{Res}_{z=\xi} F^{*}(z) .
$$

If follows that if $\psi(x)$ is stepwise constant in an interval $(\alpha, \beta)$, then so is $\psi^{*}(x)$ (and $\psi_{1}(x)$ since a similar procedure clearly applies to 
$\left.F_{1}(z)\right)$. Moreover, if $c \notin[A, B]$, say $B<c<\infty$, then $b<b^{*}$ by Theorem 2 and $x_{n, n-1}^{*}<b, \lim _{n \rightarrow \infty} x_{n, n}^{*}=b^{*}$. Since every point of increase of $\psi^{*}(x)$ is a limit point of zeros of $\left\{P_{n}^{*}(x)\right\}$ [5], it follows that $\psi^{*}(x)$ is constant for $b<x<b^{*}$, hence $\psi^{*}(x)$ must have a jump at $b^{*}$ which can be determined by (4.5).

5. Examples. The Tchebicheff polynomials furnish simple examples for which $P_{n}^{*}(x)$ can be determined explicitly and for which $\psi^{*}(x)$ is of "mixed type," that is, $\psi^{*}(x)$ is absolutely continuous on part of the orthogonality interval and is a step function (with a single jump) on the remainder.

(a) $P_{n}(x)=2^{-n} U_{n}(x)=2^{-n} \sin (n+1) \theta / \sin \theta, x=\cos \theta$. We have $b_{1}=b_{n}=0, \lambda_{n}=1 / 4(n \geqq 2) ; F(z)=2 /\left(z+\left(z^{2}-1\right)^{1 / 2}\right)$ (cf. [3]). Clearly ${ }^{2}$ $Q_{n}(x)=P_{n}(x)$ so by (2.1), (4.1) and (4.4),

$$
P_{n}^{*}(x, c)=2^{-n}\left[U_{n}(x)-2 c U_{n-1}(x)\right]
$$

and ${ }^{3}$

$$
\psi^{*}(x)-\psi^{*}\left(x_{0}\right)=\frac{2}{\pi} \int_{x_{0}}^{x} \frac{\left(1-t^{2}\right)^{1 / 2}}{4 c^{2}+1-4 c t} d t, \quad-1<x_{0}<x<1 .
$$

Here $-A=B=1 / 2$ so $\psi^{*}(x)$ is constant for all $x \notin(-1,1)$ except for $|c|>1 / 2$ when it has a jump at $\xi=c+1 / 4 c$ which is found by (4.5) to be $1-1 / 4 c^{2} \cdot \psi^{*}(x)$ is continuous at \pm 1 except possibly when $c$ $= \pm 1 / 2$. However, in this case, $P_{n}^{*}(x, c)$ reduces to a Jacobi polynomial:

$$
P_{n}^{*}(x, c)=2^{n}(n !)^{2} /(2 n) ! P_{n}^{(c,-c)}(x), \quad c= \pm 1 / 2 .
$$

(b) $P_{n}(x)=2^{1-n} T_{n}(x)=2^{1-n} \cos n \theta$. Here $b_{n}=0(n \geqq 1), \lambda_{n}=1 / 4$ for $n \geqq 3$ but $\lambda_{2}=1 / 2 ; F(z)=\left(z^{2}-1\right)^{-1 / 2}[3]$. Thus $Q_{n}(x)=2^{-n} U_{n}(x)$ and we have

$$
\begin{aligned}
P_{n}^{*}(x, c) & =2^{1-n}\left[T_{n}(x)-c U_{n-1}(x)\right], \\
\psi^{*}(x)-\psi^{*}\left(x_{0}\right) & =\frac{1}{\pi} \int_{x_{0}}^{x} \frac{\left(1-t^{2}\right)^{1 / 2}}{1+c^{2}-t} d t, \quad-1<x_{0}<x<1 .
\end{aligned}
$$

$A=B=0$, hence for all $c \neq 0, \psi^{*}(x)$ is continuous at \pm 1 and constant for $x \notin(-1,1)$ except for a jump at $\xi=(\operatorname{sgn} c)\left(1+c^{2}\right)^{1 / 2}$ of magnitude $|c|\left(1+c^{2}\right)^{-1 / 2}$.

${ }^{2}$ Sherman has shown that this is essentially the only case when $Q_{n}(x)=P_{n}(x)$, as is also evident from the recurrence formula.

3 The constant factor is determined in accordance with $\int_{-\infty}^{\infty} d \psi^{*}(x)=\lambda_{1}=1$. 
The modified Lommel polynomials, $P_{n}(x)=\left[2^{n}(\nu)_{n}\right]^{-1} h_{n, \nu}(x)$ (cf. [1]) furnish another simple example where $P_{n}^{*}(x)$ can be explicitly given. Since $b_{1}=b_{n}=0, \lambda_{n} \equiv \lambda_{n, \nu}=[4(n+\nu-1)(n+\nu-2)]^{-1}=\lambda_{n-1, \nu+1}$ $(n \geqq 2)$, we have $Q_{n}(x)=\left[2^{n}(\nu+1)_{n}\right]^{-1} h_{n, \nu+1}(x), P_{n}^{*}(x, c)=\left[2^{n}(\nu)_{n}\right]^{-1}$ - $\left[h_{n, \nu}(x)-2 c \nu h_{n-1, \nu+1}(x)\right] . \psi^{*}(x)$ is a step function whose jumps can be obtained from (4.1) and (4.5) with $F(z)=J_{\nu}(1 / z) / J_{\nu-1}(1 / z)$ where $J_{\nu}(z)$ is the Bessel function of order $\nu$.

REMARK. It may be noted that in the preceding examples, $P_{n}^{*}(x, c)$ satisfies Dickinson's relation (1.2) (since $b_{n}=0(n \geqq 1)$ ).

\section{REFERENCES}

1. D. Dickinson, On Lommel and Bessel polynomials, Proc. Amer. Math. Soc. vol. 5 (1954) pp. 946-956.

2. J. Favard, Sur les polynomes de Tchebicheff, C. R. Acad. Sci. Paris vol. 200 (1935) pp. 2052-2053.

3. J. Sherman, On the numerators of the convergents of the Stieltjes continued fractions, Trans. Amer. Math. Soc. vol. 35 (1933) pp. 64-87.

4. J. Shohat and J. Sherman, On the numerators of the continued fraction . . , , Proc. National Acad. Sci. U.S.A. vol. 18 (1932) pp. 283-287.

5. J. Shohat and J. Tamarkin, The problem of moments, Amer. Math. Soc. Mathematical Surveys no. 1, 1943.

6. H. S. Wall, Analytic theory of continued fractions, Van Nostrand, 1948.

SeAtTle University 